\title{
Detection of turbulent/non-turbulent interface for an axisymmetric turbulent jet: evaluation of known criteria and proposal of a new criterion
}

\author{
Ravi Kumar Anand · B. J. Boersma • \\ Amit Agrawal
}

Received: 19 October 2008/Revised: 21 May 2009/Accepted: 21 May 2009/Published online: 7 June 2009

(C) The Author(s) 2009. This article is published with open access at Springerlink.com

\begin{abstract}
In this paper, we evaluate several criteria for the detection of turbulent/non-turbulent interface using direct numerical simulation and particle image velocimetry data of an axisymmetric turbulent jet. The possibility of identifying the interface from information available in wholefield velocity data alone is also explored. The present results using a Concentration thresholding technique compare well against available results obtained using a similar detection criterion. It is noted that Concentration and Vorticity criteria are difficult to apply with standard PIV data and therefore a new criterion based on azimuthal vorticity and streamwise velocity—quantities available from such data, is proposed. The proposed criterion scores over previously employed criteria in terms of its simplicity of evaluation, and can possibly be applied to other flows not tested here. The instantaneous location of the interface as detected from the different criteria differs substantially. However, the conditionally averaged streamwise velocity, azimuthal vorticity, and Reynolds shear stress across the interface obtained from the new criterion, as well as from the previous criteria, agree reasonably well against available results. The present work further suggests that different criteria, even with slightly sub-optimal threshold value, can provide quantitatively similar ensemble-averaged results.
\end{abstract}

\footnotetext{
R. K. Anand · A. Agrawal $(\bowtie)$

Department of Mechanical Engineering,

Indian Institute of Technology Bombay,

Powai, Mumbai 400076, India

e-mail: amit.agrawal@me.iitb.ac.in; amit.agrawal@iitb.ac.in

B. J. Boersma

Laboratory for Aero en Hydrodynamics,

Delft University of Technology, Leeghwaterstraat 21,

2628 CA Delft, The Netherlands
}

\section{Introduction}

With the widespread use of techniques such as particle image velocimetry (PIV) and direct numerical simulation (DNS), wholefield velocity information of a flow in the region of interest is readily available (Adrian 2005; Prasad 2000). With this spatially resolved velocity data, it should be possible to examine flows in greater detail and explore features that are not accessible with the earlier point-wise measurement techniques (Agrawal and Prasad 2002, 2003a). Further, the data are quantitative in nature, unlike flow visualization techniques which provide only qualitative information (Prasad and Sreenivasan 1989; Agrawal et al. 2004). In this paper, we explore the possibility of detecting an important characteristic of turbulent flows, the turbulent/ non-turbulent interface, which has not received much attention primarily because of its irregular and time-varying shape and position, making it difficult to study. The attempt here is to use information which is generally available from wholefield velocity data such as velocity and out-of-plane vorticity and without taking recourse to any other input.

A thin and highly irregular interface, with a thickness of about one-two orders of magnitude smaller than the integral scale (Chevray 1982; Bisset et al. 2002; Hunt et al. 2006; Holzner et al. 2008), is known to separate the turbulent motion from the surrounding flow. The two regions can be readily identified by adding dye to one region and using laser or some other light source for illumination (Dimotakis et al. 1983; Westerweel et al. 2002). Prasad and Sreenivasan (1989) used thresholding to determine the scalar interface of an axisymmetric turbulent jet. In general, the interface is known to be wrinkled and spread over a wide range of small scales, particularly at high Reynolds numbers; these small scales are self similar in structure and can be well described as fractal (Sreenivasan et al. 1989). 
However, it is not easy to separate out the two regions when no marker is present in the flow. There is, however, a rapid change in the magnitude of vorticity across the turbulent/non-turbulent interface, which can be used to advantage. Furthermore, the flow in the non-turbulent region is practically irrotational and is rotational in the turbulent region (Corrsin 1943; Corrsin and Kistler 1954; see also Pope 2000, p 167-173). This property has been used by Bisset et al. (2002), Mathew and Basu (2002) and is further tested in the present paper for interface detection. The former authors in their analysis of DNS data for a plane wake required that the magnitude of normalized vorticity at the interface be equal to a pre-determined threshold. Mathew and Basu (2002) verified the above criteria to be satisfactory, with a similar value of threshold, in their DNS study of a temporal circular shear flow. Note that such a criterion can be used with DNS data because of the availability of all three components of vorticity, which is usually not the case with experimental data.

Westerweel et al. (2002, 2005) and Holzner et al. (2006) employed a novel method of performing PIV and flow visualization (LIF) concurrently on an axisymmetric turbulent-free jet and oscillating grid, respectively, to overcome this difficulty. While a threshold in concentration provided by the LIF data was used for interface detection, the PIV data were used to obtain conditionally averaged quantities across the interface. However, the approach of Westerweel et al. (2002) is somewhat cumbersome in that two synchronized cameras viewing the same area and preferably positioned on opposite sides of the test section have to be employed. The noise content in the measurements and the finite resolution of PIV further complicates matters. Nonetheless, the technique has been utilized by Holzner et al. (2007, 2008) to study the process of entrainment and the role of small scale eddies. Da Silva and Pereira (2008) have computed the invariants of the velocity gradient, rate-of-strain, and rotation tensors across the turbulent/non-turbulent interface in an effort to understand the process of turbulent entrainment better. Note that the Schmidt number of the dye is much greater than unity in these studies. One important observation of their study is that nibbling by the small eddies contributes substantially to the total fluid entrained, as suggested by the earlier studies of Mathew and Basu (2002), Agrawal and Prasad (2004), and Westerweel et al. (2005).

Prior to the advent of wholefield measurement techniques, precise determination of interface location was difficult and mostly the intermittency function (measuring the probability of the flow being turbulent) was reported (see example, Kibens et al. 1974; Chevray 1982). The generation of the intermittency function, however, suffers from a certain amount of arbitrariness and may not detect the non-turbulent events occurring in long-duration turbulent events well; along with these it has several other difficulties (Antonia 1981). Using a passive scalar (such as temperature) was found to give better results as compared to either velocity or vorticity, and has been employed by LaRue (1974), LaRue and Libby (1974) and Antonia et al. (1975), among others.

In this paper, a quantitative comparison of three conditionally averaged quantities-streamwise velocity, azimuthal vorticity, and Reynolds shear stress-across the interface, is undertaken. These quantities (appropriately normalized) are obtained from the Concentration, Vorticity, and Velocity detection criteria. Although some comparison of the different detection criteria is provided in Holzner et al. (2006), our study differs from them in two ways: First, the availability of three-dimensional data in the present case is an advantage as it allows us to compute, for example, the total vorticity. Second, we have chosen a flow for which results are available in the literature. A novel algorithm which can be employed to determine the location of the turbulent/non-turbulent interface from wholefield velocity data, without requiring any additional input, is also proposed and evaluated. Further, a discussion on choosing the threshold value is provided. The literature survey suggests that this is the first attempt to develop such a criterion.

\section{Methodology}

Both numerical and experimental data have been analyzed in this work. A brief introduction to these is presented in this section.

\subsection{Direct numerical simulation (DNS)}

The same code as developed by Boersma et al. (1998), Lubbers et al. (2001) and used earlier by Agrawal et al. (2005), has been employed for the present work. The simulations are performed on a spherical grid of size $270 \times 80 \times 48$ in $r, \theta, \phi$ directions, respectively, for a jet. The computational domain is a conical volume segment of spherical shell which spans between 50 and 93 nozzle diameters in the streamwise direction as measured from the origin of the spherical coordinate system. The lateral edge of the domain is angled at $\pi / 40$ so as to match the spreading rate of the jet. The scalar field is discretized with the total variation diminishing scheme and second order Adams-Bashforth method is used for time integration. The inflow, outflow, and lateral boundary conditions are kept similar to that used in the earlier works. The exit Reynolds number $\left(=U_{0} d / v\right.$, where $U_{0}$ is the exit velocity, $d$ is the orifice diameter, and $v$ is the kinematic viscosity of the fluid) is 1000 and Schmidt number $(=v / D$ where $D$ is the molecular diffusivity of the dye) is unity, in the present 
simulations. The current simulations are able to resolve the Kolmogorov length and concentration scale sufficiently (Boersma et al. 1998; Agrawal et al. 2005). The data set comprised 37 time frames taken after the jet had become stationary. A fortran code is developed to automate the detection procedure, as discussed in detail in Sect. 3.5.

\subsection{Particle image velocimetry measurements (PIV)}

The PIV data of Agrawal and Prasad (2002, 2003a) have been reanalyzed in this work. The experiments are performed on a water jet issuing from an orifice 2-mm diameter with the PIV view-frame located between $110 \leq x / d \leq 175$, where $x$ is the streamwise coordinate. The exit Reynolds number of the flow is 3000 . The vectors are spaced $2 \mathrm{~mm}$ apart and all scales bigger than the Taylor microscales are being resolved in the measurements (Agrawal 2005). The accuracy of the measurements is 1/10th of a pixel (Agrawal and Prasad 2002). The noise level of velocity is estimated to be of the order of $1 \mathrm{~mm} / \mathrm{s}$. The dataset consists of 126 PIV frames. It was verified that the flow satisfies the self-similarity condition (Agrawal and Prasad 2002, 2004). The detection procedure of the interface for the PIV data is similar to DNS data.

\section{Algorithm for detection of interface}

A brief discussion on the algorithm for detecting the interface from different criteria used in this paper is provided in this section. A discussion on the threshold value employed for each criterion is also presented.

\subsection{Concentration criterion}

The instantaneous DNS data are marked as per the following condition:

$$
\begin{aligned}
\frac{C_{\text {ins }}}{C_{0}} & >0.03 \text { flow in turbulent region } \\
& \leq 0.03 \text { otherwise }
\end{aligned}
$$

where $C_{\text {ins }}$ is the instantaneous scalar concentration of the jet fluid and $C_{0}$ is the scalar concentration at the nozzle exit. See Sect. 3.6 for procedure to obtain the threshold value. This criterion is termed as "Concentration criterion" in this paper. (Note that when referring to criterion, Concentration is capitalized.) Normalization of instantaneous concentration by its nozzle exit value employed here is similar to that of Holzner et al. (2006), where the maximum concentration, obtained from a view frame of $200 \times 100 \mathrm{~mm}^{2}$ extending over the entire tank, was used for normalization. Normalization by average centerline concentration $\left(C_{\mathrm{c}}\right)$ value is also possible; the threshold value then changes to $\frac{0.03 C_{0}}{C_{\mathrm{c}}}$.

\subsection{Vorticity criterion}

The instantaneous data of the jet fluid are checked for the following condition:

$$
\begin{aligned}
\left(\frac{\omega_{\text {tot }} b}{U_{\mathrm{c}}}\right) & >0.1 \text { flow in turbulent region } \\
& \leq 0.1 \text { otherwise }
\end{aligned}
$$

where $\omega_{\text {tot }}=\sqrt{\left(\omega_{x}^{2}+\omega_{y}^{2}+\omega_{z}^{2}\right)}$ is the total magnitude of the vorticity at any location of the jet fluid, $U_{\mathrm{c}}$ is the mean streamwise velocity, and $b$ is the velocity width (radial distance at which streamwise velocity becomes $1 / e$ of the centerline velocity). This criterion is termed as "Vorticity criterion" in this paper.

\subsection{Velocity criterion}

Similar to the Concentration criterion, this criterion checks for the instantaneous streamwise velocity $\left(U_{\text {ins }}\right)$ of the jet as follows:

$\frac{U_{\text {ins }}}{U_{0}}>0.03$ flow in turbulent region

$$
\leq 0.03 \text { otherwise }
$$

where $U_{0}$ is the streamwise velocity at the nozzle exit. This criterion is termed as "Velocity criterion" in this paper. It should be noted that unlike vorticity (which is ideally zero and non-zero in the non-turbulent and turbulent regions, respectively), the velocity may be non-zero in the nonturbulent region. This is attributed to the presence of velocity induced by the vortical structures in the turbulent region. The threshold value needs to be carefully chosen such that it is well above the induced velocity fluctuations. The threshold value (0.03) satisfies this condition, as shown in Fig. 1b presented later.

\subsection{New (Velocity-Vorticity) criterion}

This criterion computes the azimuthal vorticity component from the instantaneous flow data and then marks as per the following condition:

$$
\begin{aligned}
\frac{U_{\text {ins }}}{\left(\left|\omega_{z}\right| U_{c} b\right)^{1 / 2}} & <0.03 \text { flow in turbulent region } \\
& \geq 0.03 \text { otherwise }
\end{aligned}
$$

where $\omega_{z}$ is azimuthal vorticity. This criterion is termed as "Velocity-Vorticity criterion" in this paper. The proposed criterion is somewhat equivalent to satisfying the double 

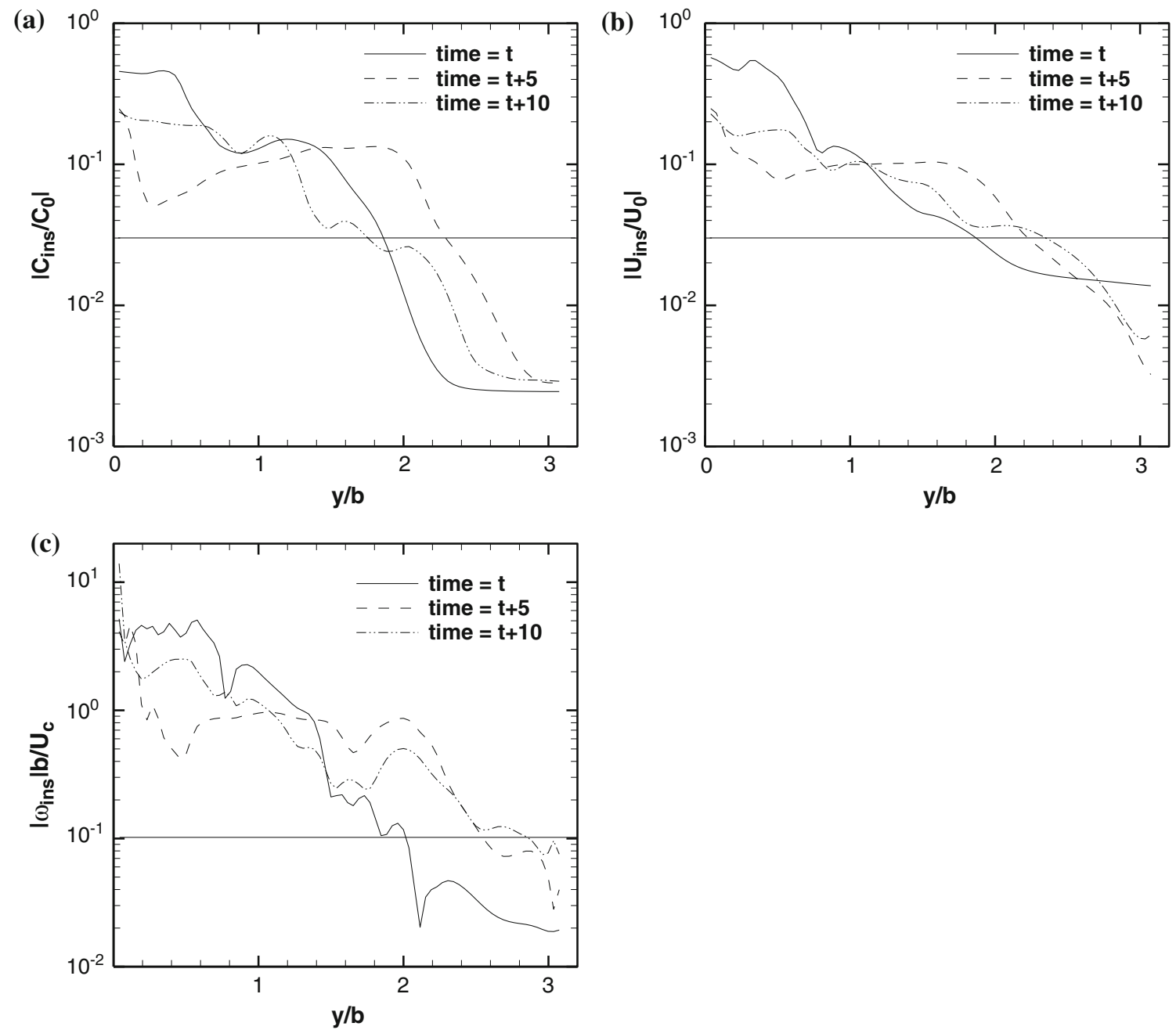

Fig. 1 Radial profiles of (a) scalar concentration, (b) velocity and (c) vorticity, at different time instances for the DNS results at axial location $z / d=22.3$

criteria of normalized velocity and azimuthal vorticity simultaneously.

\subsection{Location of interface}

To locate the interface, a fortran code is developed for each of the above criteria. The code checks for the interface starting from the jet centerline and moving radially outwards. The radial position (for each axial location) where the condition given in Eqs. 1, 3, 5 or 7 is first violated is marked as the interface for that axial location. Note that the code does not check for multiple radial locations at which the above condition can be violated (as would occur, for example, with a detrained fluid blob not connected to the main jet body). Also, no explicit effort to maintain continuity of the interface has been made in the present work.

\subsection{Determination of threshold value}

The basis for choosing the threshold values in Eqs. 1-8 is discussed in this subsection. The threshold values are evaluated using the following two approaches: in the first approach, the procedure of Prasad and Sreenivasan (1989) is employed. For this, the average concentration of all pixels above an arbitrary chosen threshold value is plotted as a function of the threshold value. The plot (not shown here) reveals that there are two regions with different slopes. The slope of the curve changes at a value of around 0.03 . This value $(0.03)$, interestingly matches with that of Westerweel et al. (2002), and has therefore been chosen as the threshold value in Eqs. 1 and 2.

The second procedure for obtaining the threshold value is as follows: a threshold is first arbitrarily chosen and interface corresponding to this threshold value is obtained. 
The root mean square (RMS) difference of this interface location from that obtained from the Concentration criterion $\left(\frac{C_{\text {ins }}}{C_{0}}=0.03\right)$ is noted. The reason for choosing this difference-against-Concentration criterion is that results are available for this criterion in a turbulent jet (this point is discussed further in Sect. 6.1). The threshold value at which the RMS difference is minimized is regarded as the optimal value. This procedure has been employed with Vorticity, Velocity, and Velocity-Vorticity criteria. Table 1 presents the RMS difference as a function of threshold values for these three criteria. For instance, as depicted in Table 1, the minimum RMS difference for Vorticity criterion occurs at a threshold of 0.10 ; therefore, a threshold value of 0.10 has been taken for this criterion. Note that the same threshold value (of 0.10) has been suggested by Mathew and Basu (2002) for a turbulent circular temporal shear layer. However, this threshold value is lower than that used by Bisset et al. (2002) $(=0.7)$ for a turbulent planar wake, owing to difference in the flow. Again, Table 1 shows that the RMS difference is minimum at a threshold value of 0.03 for both Velocity and VelocityVorticity criteria. Therefore, threshold value of 0.03 is taken for detecting the interface with these two criteria.

The threshold value obtained from the second method was compared against that from the first method for the Velocity criterion, and the two agreed. A sensitivity of the results on the threshold value employed is presented later in Sect. 6.2.

The threshold value of 0.03 obtained for Velocity criterion is coincidentally equal to the threshold value used for Concentration criterion. It might be tempting to attribute this to the Schmidt number being unity in the simulations. For comparison, in the experimental work of Holzner et al. (2006), the Schmidt number is 2000, and the threshold levels are 0.23 and 0.20 for the Concentration and Velocity criteria, respectively. Thus, the Schmidt

Table 1 RMS difference in the interface location at different threshold values using different criteria

\begin{tabular}{|c|c|c|c|}
\hline \multirow{2}{*}{$\begin{array}{l}\text { Threshold } \\
\text { value }\end{array}$} & \multicolumn{3}{|c|}{ RMS difference/velocity width } \\
\hline & $\begin{array}{l}\text { Concentration } \\
\text { criterion-Vorticity } \\
\text { criterion }\end{array}$ & $\begin{array}{l}\text { Concentration } \\
\text { criterion-Velocity } \\
\text { criterion }\end{array}$ & $\begin{array}{l}\text { Concentration } \\
\text { criterion-Velocity- } \\
\text { Vorticity criterion }\end{array}$ \\
\hline 0.01 & 1.573 & 0.604 & 1.210 \\
\hline 0.02 & 1.329 & 0.532 & 1.054 \\
\hline 0.03 & 1.170 & 0.514 & 0.995 \\
\hline 0.05 & 0.974 & 0.635 & 1.010 \\
\hline 0.07 & 0.849 & 0.749 & 1.043 \\
\hline 0.10 & 0.734 & 0.917 & 1.072 \\
\hline 0.15 & 0.779 & 1.161 & 1.075 \\
\hline 0.20 & 0.792 & 1.367 & 1.082 \\
\hline 0.25 & 0.844 & 1.534 & 1.121 \\
\hline
\end{tabular}

number does not appear to be responsible for the same threshold value in Concentration and Velocity criteria.

We now present radial variation of instantaneous absolute concentration, streamwise velocity, and total vorticity (Fig. 1a-c) for the DNS data, at a few arbitrary instants. The corresponding threshold values are also marked in the figure, which suggests that the threshold value is well above the noise level in all cases. For example, with streamwise velocity, the noise level is about $10^{-3}$ which is much lower then the threshold value of 0.03 .

\section{Comparison of known criteria}

The results for variation of average quantities across the interface as obtained from the DNS data of an axisymmetric turbulent jet are presented in this section. The purpose is to compare the two commonly used criteria-namely, Vorticity and Concentration. A quantitative comparison of results from different criteria is difficult to find in the literature (Holzner et al. 2006). The results are compared against those of Westerweel et al. (2005). This comparison will be useful while evaluating the new criteria proposed in Sect. 5.

\subsection{Concentration criterion}

In this subsection, the results obtained from the Concentration criterion are presented. Note that this criterion has been employed earlier, for example by Prasad and Sreenivasan (1989) and Westerweel et al. (2002, 2005). Whereas the earlier results are experimental, the results presented in this section are numerical. Also, there are differences in the values of the governing parameters as noted in Table 2. In particular, the Schmidt number is unity in the simulations which is much less than that in the experiments. A direct comparison of the conditionally averaged profiles for the streamwise velocity, azimuthal vorticity, and Reynolds shear stress $(-\bar{u} \bar{v})$, across the interface against the results of Westerweel et al. (2005) is shown in Figs. 2, 3, and 4. (Note that results from several criteria are included in the figures for ease in comparison and compactness. A

Table 2 Value of different parameters employed in the experimental and DNS study

\begin{tabular}{lcc}
\hline Source & $R e$ & $S c$ \\
\hline PIV data & 3000 & - \\
DNS data & 1000 & 1 \\
Westerweel et al. (2005) & 2000 & 2,000 \\
\hline $\begin{array}{l}\text { The values of Westerweel et al. (2005) is also included for } \\
\text { comparison }\end{array}$ &
\end{tabular}




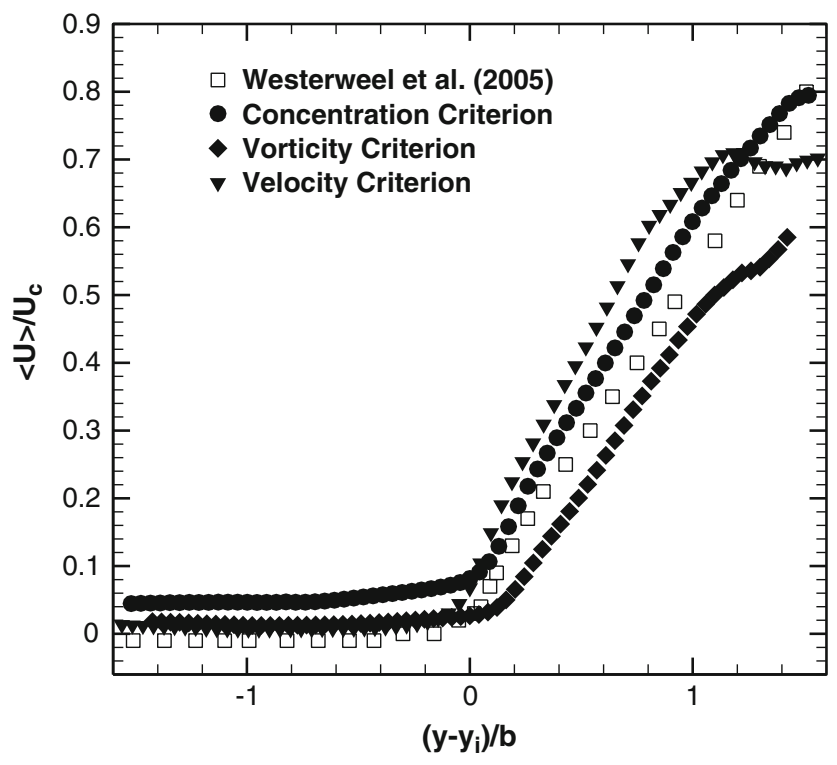

Fig. 2 Conditionally averaged streamwise velocity profile across the interface using three different criteria obtained from DNS data. The data of Westerweel et al. (2005) are also shown for comparison

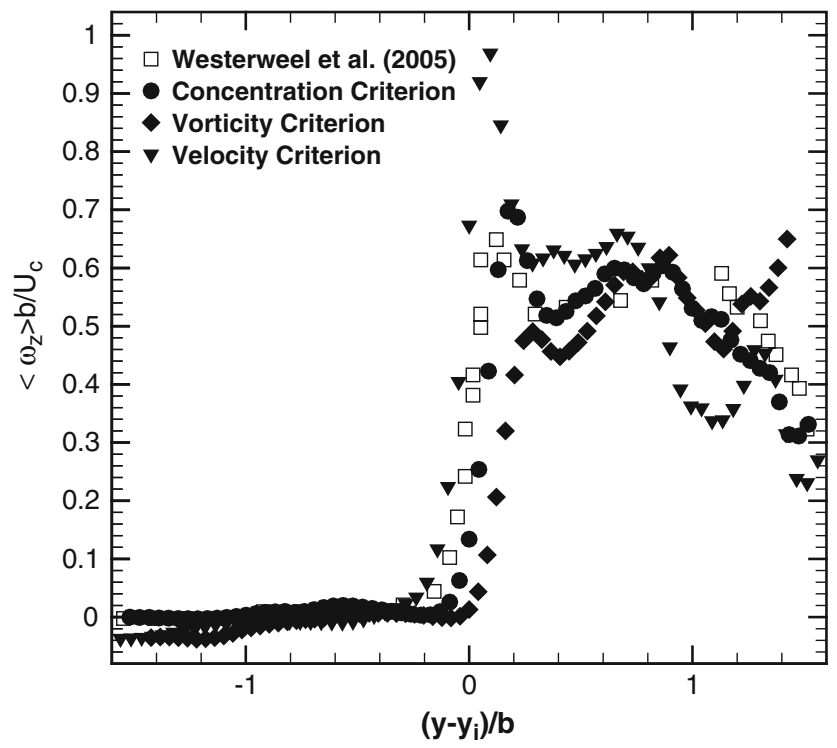

Fig. 3 Conditionally averaged normalized vorticity across the interface using three different criteria obtained from DNS data. The data of Westerweel et al. (2005) are also shown for comparison. Note that the data in Westerweel et al. (2005) have more points in this figure than in the original data

discussion of results from other criteria is presented in later sections.)

It is observed from Fig. 2 that the normalized streamwise velocity computed from the numerical simulations agrees within $8 \%$ in the turbulent region with the experimentally obtained value. Note that in the figure, $y$ denotes the radial coordinate and $y_{i}$ is the instantaneous position of

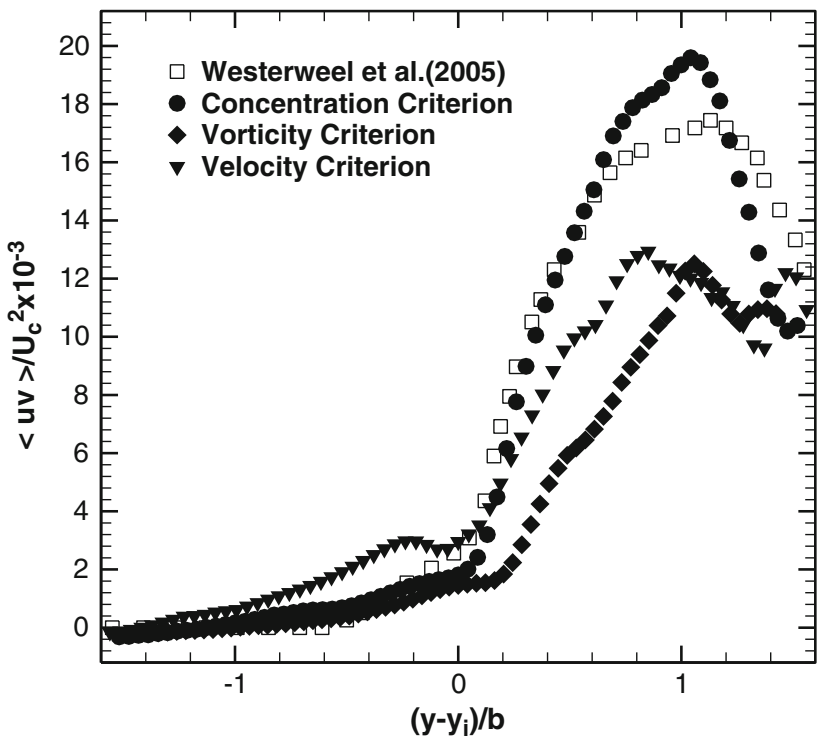

Fig. 4 Conditionally averaged normalized Reynolds stress shear profile across the interface using three different criteria obtained from DNS data. The data of Westerweel et al. (2005) are also shown for comparison

the interface. Figure 3 compares the conditionally averaged normalized azimuthal vorticity where normalization is done with the velocity width and mean centerline velocity of the jet. The match of the vorticity profile against Westerweel et al. (2005) is quite encouraging in both the turbulent and non-turbulent regions. The vorticity remains constant for almost one jet width in the turbulent region and then starts to fall as it reaches near the jet centerline. The conditionally averaged Reynolds shear stress is shown in Fig. 4. The Reynolds stress is found to be increasing steadily from the -1.6 radial location in the irrotational region and is similar to the profile by Westerweel et al. (2005) in the turbulent region, except when $\left(y-y_{i}\right) / b$ is close to unity.

Both vorticity (Fig. 3) and Reynolds shear stress (Fig. 4) across the interface agree well between experiments and simulations; noticeable differences are, however, obtained for $\left(y-y_{i}\right) / b>0.6$. The above results establish confidence in the data-processing algorithms employed in the present work, besides reinforcing the earlier results of Westerweel et al. (2002, 2005). These results also suggest that results from a new criterion should be compared in the range $\left(y-y_{i}\right) / b<0.6$ while differences (due to factors not clear to the authors) may occur at larger radial locations.

\subsection{Vorticity criterion}

The Vorticity criterion employed here was suggested by Bisset et al. (2002) and Mathew and Basu (2002). 
However, the threshold value to be employed in Eqs. 3 and 4 for an axisymmetric turbulent jet is not available; hence we evaluate it through a systematic variation of the threshold value, as discussed in Sect. 3.6.

The conditionally averaged quantities across the interface as obtained from the Vorticity criterion are also included in Figs. 2, 3, and 4. The conditionally averaged streamwise velocity increases monotonically in the turbulent region (Fig. 2). The rise in velocity across the interface and in the turbulent region is, however, less sharp as compared to the earlier result of Westerweel et al. (2005). The value obtained is therefore about $10 \%$ lower for $0<\left(y-y_{i}\right) / b<0.9$, and differs substantially beyond it. Fig. 3 shows that the vorticity is almost zero in the irrotational region and non-zero in the turbulent region. The rise across the interface is not that steep and the overshoot is much weaker, with the Vorticity criterion. The vorticity in the turbulent region is comparable with the results of Westerweel et al. (2005) in the region $\left(y-y_{i}\right) / b<1.2$ and differs noticeably beyond it. In particular, a rising trend in vorticity is obtained near the centerline. The Reynolds shear stress as obtained from the Vorticity criterion although qualitatively similar to Westerweel et al. (2005) is almost $30 \%$ smaller than that obtained from the Concentration criterion in the turbulent region (Fig. 4). A slower rise across the interface with the Vorticity criterion as compared to the earlier result is again noted.

The above results suggest some differences (in particular for the Reynolds stress) in the conditionally averaged quantities as obtained from the Concentration and Vorticity criteria. Note that these results are based on the total vorticity; the results get worse when only azimuthal vorticity is employed for interface detection, suggesting that azimuthal vorticity alone would be inadequate to mark the interface. As will be discussed in Sect. 6.1, the discrepancy is due to the Vorticity criterion's interface lying mostly outside (away from the jet centerline) with respect to the Concentration criterion's interface.

\section{New interface detection criteria}

As discussed in Sect. 1, employing either Concentration or Vorticity criteria with experimentally obtained wholefield velocity data is difficult. In this section, we propose and evaluate two alternate criteria which are relatively simple to apply with such data.

\subsection{Velocity criterion}

The straightforward approach of thresholding the streamwise velocity for detection of the interface is explored first. The fluid is in the turbulent region if the streamwise velocity is greater than a threshold and in the non-turbulent region otherwise (see Sect. 3.3). This approach has been employed earlier by Holzner et al. (2006); in their study the threshold was set as four times the noise level in an undisturbed flow. Here, we compute the RMS difference to determine the value of the threshold as discussed Sect. 3.6. A threshold of 0.03 is obtained and employed for results presented in this section.

The results obtained for the conditionally averaged streamwise velocity, azimuthal vorticity, and Reynolds shear stress across the interface are again included in Figs. 2, 3, and 4. The conditionally averaged streamwise velocity is zero in the irrotational region with this criterion, and it increases monotonically in the turbulent region (Fig. 2). However, the Velocity criterion over-predicts the velocity (relative to Concentration criterion) in the turbulent region, other than near the jet centerline. The difference in velocity in the turbulent region is up to $28 \%$. Note that the velocity is less than 0.03 in the non-turbulent region owing to cancelation of positive and negative velocities present outside the interface. The vorticity is observed to be zero in the irrotational region and exhibits an overshoot across the interface. However, the rise starts somewhat earlier and the overshoot at the interface is much larger than the earlier result of Westerweel et al. (2005). The vorticity value remains larger than the benchmark result for $\left(y-y_{i}\right) / b<1$, and becomes less towards the jet centerline. The Velocity criterion gives better results for Reynolds stress than the Vorticity criterion but worse than the Concentration criterion (Fig. 4). For instance, the difference with respect to Westerweel et al. (2005) is still large (upto $45 \%$ at $y=y_{i}$ and $24 \%$ in the turbulent region). Furthermore, an earlier rise in Reynolds stress is noticed on moving from non-turbulent to turbulent region for this criterion.

These results suggest that the location of the interface detected could possibly be more toward the jet centerline as compared to the Concentration interface. This point is discussed further through Figs. 11 and 12 presented later. The Velocity criterion does not seem to describe the streamwise velocity well. This motivates us to look for an alternate interface detection criterion.

\subsection{Proposal of a new (Velocity-Vorticity) criterion}

It is postulated that a detection criteria could be constructed based on streamwise velocity and azimuthal vorticity. The proposed Velocity-Vorticity criterion takes the following form:

$\frac{U}{\left|\omega_{z}\right|^{1 / 2}}<$ threshold flow in turbulent region

$\geq$ threshold otherwise 
Equations 9 and 10 when normalized appropriately take the form of equations 7 and 8 , respectively. The motivation for taking the square-root of vorticity in the above equation is that, in the self-similar regime of an axisymmetric turbulent jet, the mean vorticity varies as $\left(x-x_{0}\right)^{-2}$, whereas the mean streamwise velocity decays as $(x-$ $\left.x_{0}\right)^{-1}$ which makes the above combination of vorticity and velocity independent of the streamwise coordinate. In the above-mentioned equation, $x$ is the streamwise distance as measured from the virtual origin of the jet $\left(x_{0}\right)$. The criterion has specifically been tested on an axisymmetric turbulent-free jet but could possibly be used with other turbulent flows (with appropriate powers for velocity and vorticity) as well. Note that the present criterion involves vorticity and velocity which are associated with the small scale and large scale of turbulent motion, respectively.

\subsection{Evaluation of the Velocity-Vorticity criterion}

An instantaneous interface is presented in Fig. 5 and quantitative comparison is provided through Figs. 6, 7, and 8. Figure 5 shows a PIV frame with superimposed interface contours as detected by the Velocity-Voriticity criterion and shows both the turbulent as well as non-turbulent regions in the flow. Note that due to the finite resolution $(2 \mathrm{~mm})$ of the measurements, the detected interface appears discontinuous. Some waviness may, however, be inherent, as also evident from Fig. 10 presented later. These discontinuities cannot be avoided by using

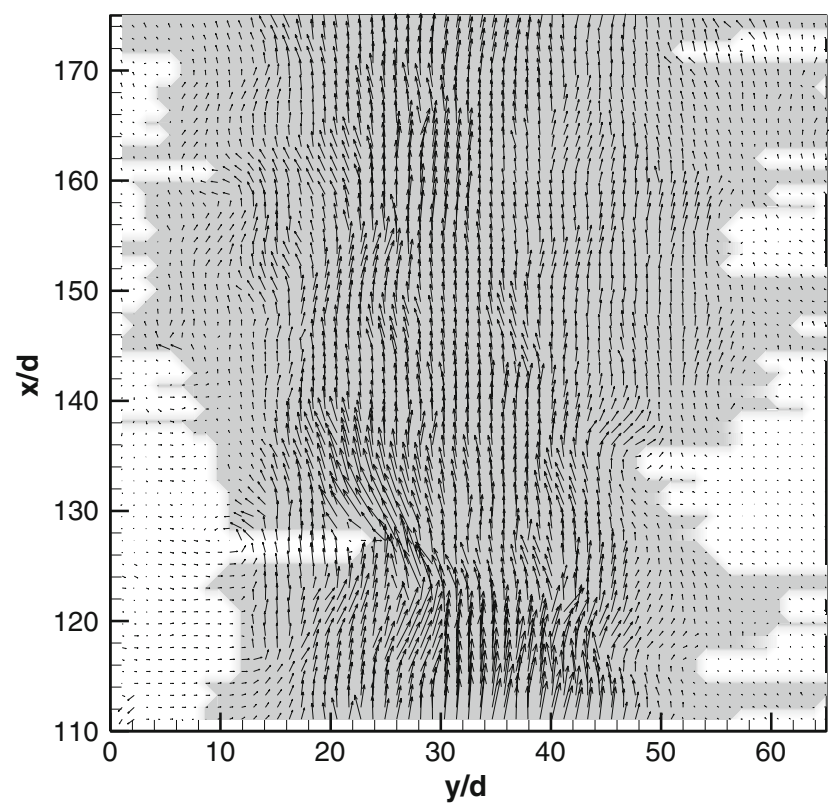

Fig. 5 Velocity vectors with superimposed interface contours as detected by the Velocity-Vorticity criterion obtained from PIV data. Here ' $d$ ' is the diameter of the orifice $(d=2 \mathrm{~mm})$

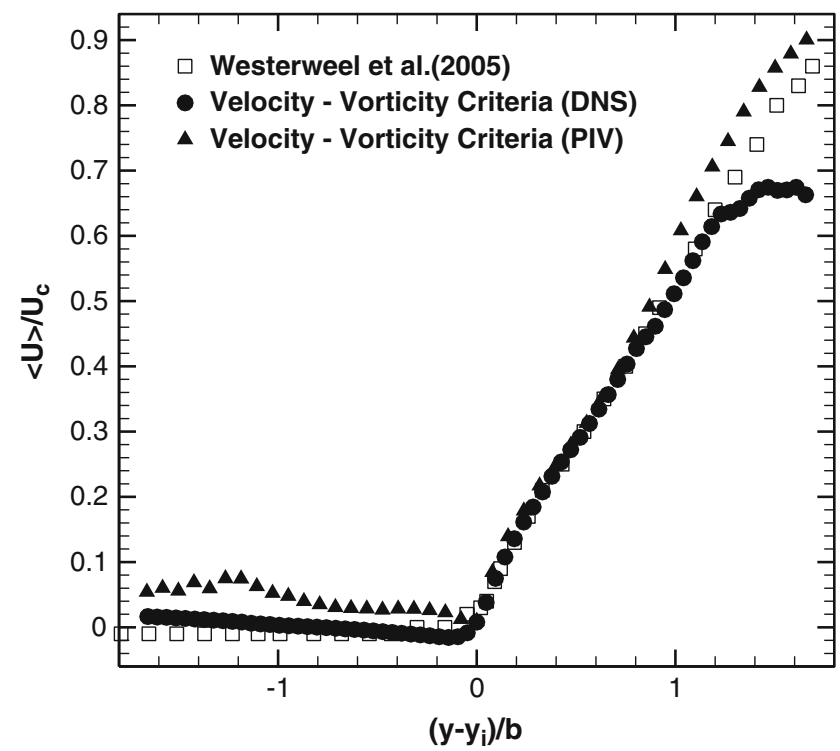

Fig. 6 Conditionally averaged streamwise velocity across the interface as obtained using the Velocity-Vorticity criterion, from both DNS and PIV data. The data of Westerweel et al. (2005) are also shown for comparison

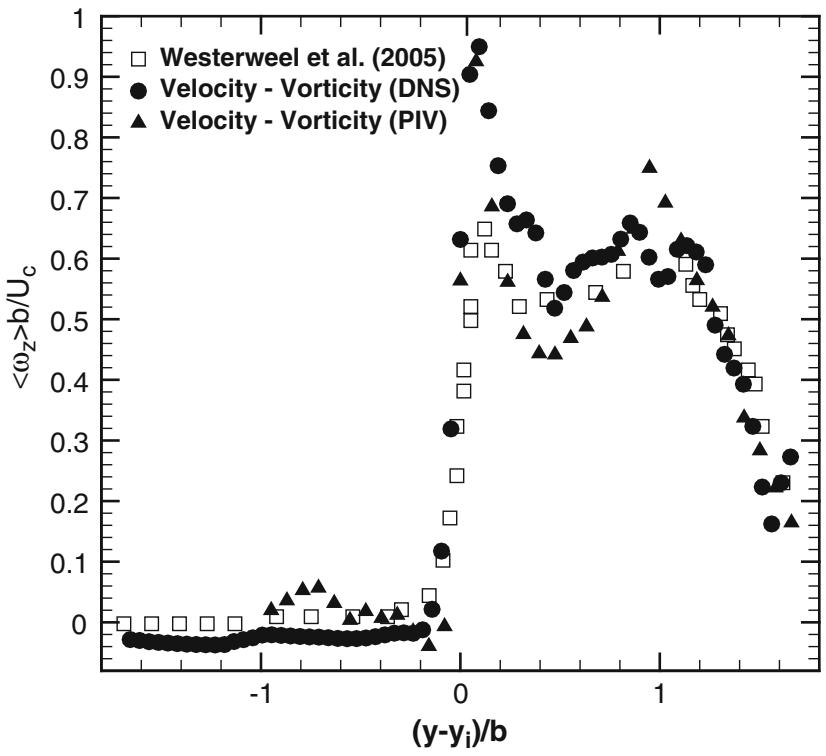

Fig. 7 Conditionally averaged normalized vorticity across the interface as obtained using the Velocity-Vorticity criterion, from both DNS and PIV data. Note that the data in Westerweel et al. (2005) have more points in this figure than in the original data

interpolated fields, as interpolation does not provide new information. Some issues associated with the quotient of two small quantities in the presence of noise are also apparent from the figure. As already stated, no explicit effort to ensure continuity of the interface has been made in this work. At some axial positions the interface is beyond the view frame- these instances are not used in the computation of the statistics. 


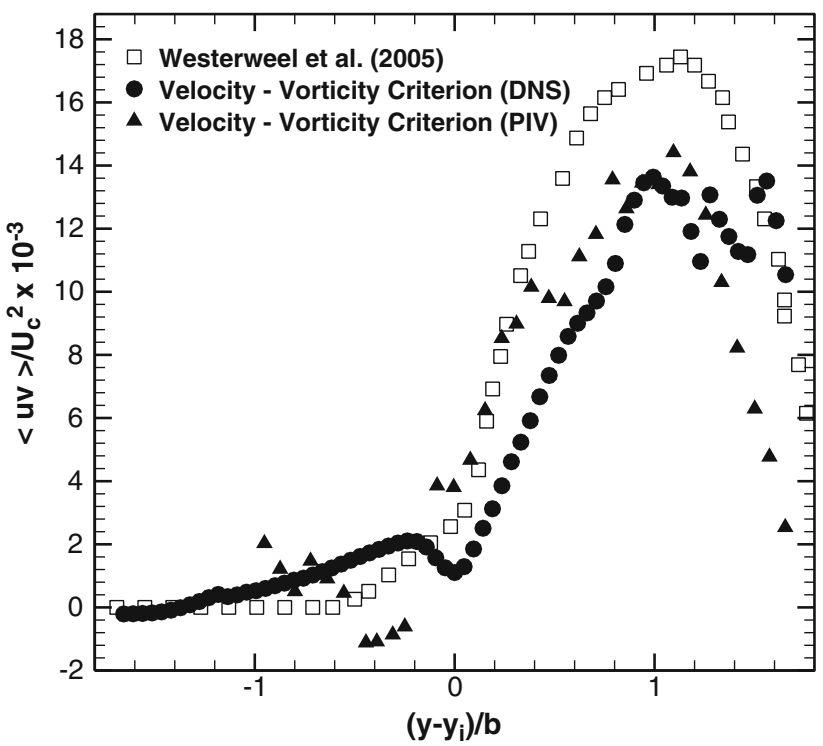

Fig. 8 Conditionally averaged normalized Reynolds shear stress across the interface as obtained using the Velocity-Vorticity criterion, from both DNS and PIV data. The data of Westerweel et al. (2005) are also shown for comparison

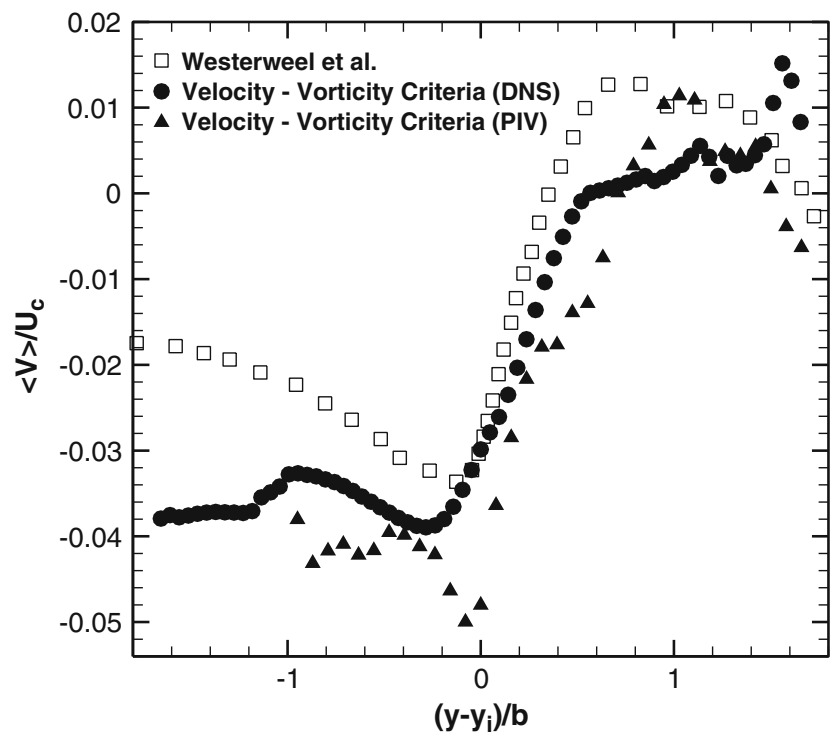

Fig. 9 Conditionally averaged normalized cross-stream velocity across the interface as obtained using Velocity-Vorticity criterion, from both DNS and PIV data. The data of Westerweel et al. are also shown for comparison

The streamwise velocity as obtained by DNS compares well with that from PIV and both of them agree with Westerweel et al. (2005) for $\left(y-y_{i}\right) / b<0.8$. Beyond it the PIV results are about 5\% higher than the benchmark result, while the DNS result is lower. Some discrepancy in results (using the same detection criterion i.e., Concentration criterion, as used earlier) with respect to the results of Westerweel et al. (2005) near the jet centerline, has already

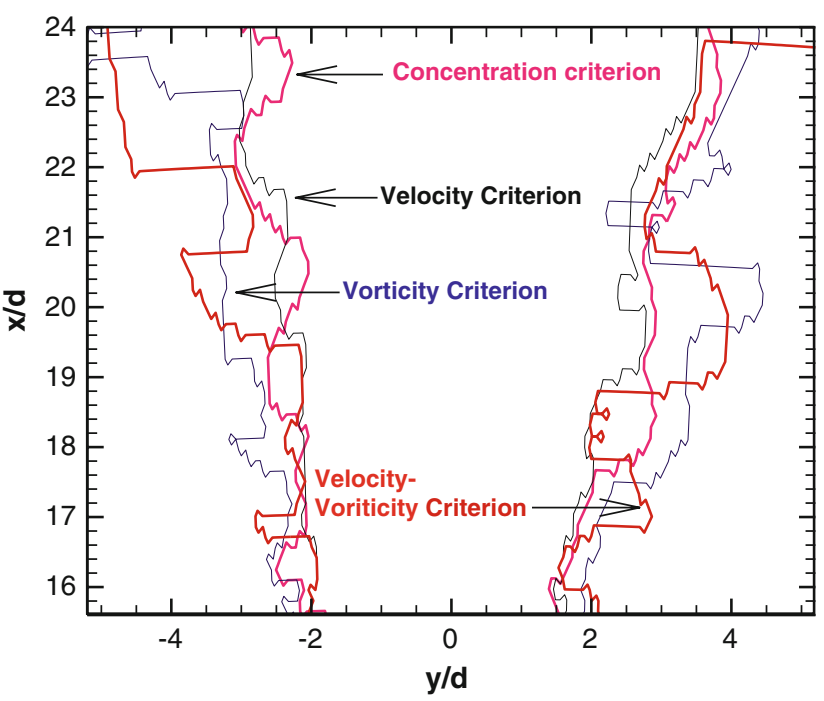

Fig. 10 Contours of interface using four different criteria tested in this study obtained from DNS data. Here " $d$ " refers to the diameter of the nozzle

been noted in Sect. 4.1. The small non-zero streamwise velocity outside the turbulent region from PIV is due to the relatively small view frame employed in these measurements.

The conditionally averaged normalized azimuthal vorticity (Fig. 7) shows a rapid change across the interface, in accord with our earlier discussion. Westerweel et al. (2005) found approximately $20 \%$ overshoot in vorticity, before it settles to a value of about 0.5 . In the present PIV data, the overshoot of vorticity is higher as compared to Westerweel et al. (2005), and the amount of overshoot agrees with the DNS result. The comparison between the three throughout the turbulent region is satisfactory. The conditionally averaged normalized Reynolds stress (Fig. 8) compares reasonably well with the result of Westerweel et al. (2005) particularly for PIV results in the turbulent region, whereas some differences are observed for the DNS results. The agreement between PIV and DNS results with the benchmark result improve closer to the jet axis.

Figure 9 shows the variation of cross-stream velocity across the interface from both DNS and PIV data of jets. The overall match of the cross-stream velocity with the data of Westerweel et al. (2002) is reasonable. Considering that the magnitude of cross-stream velocity is very small (of the order of the measurement accuracy itself; see also Agrawal and Prasad 2003b), the overall match between the three can be regarded as acceptable.

The Velocity-Vorticity criterion gives better results than both Vorticity and Velocity criteria, with respect to the quantities tested in this work. The reason for better results from the new criterion as compared to both Vorticity and Velocity criteria is explored in the next section. 


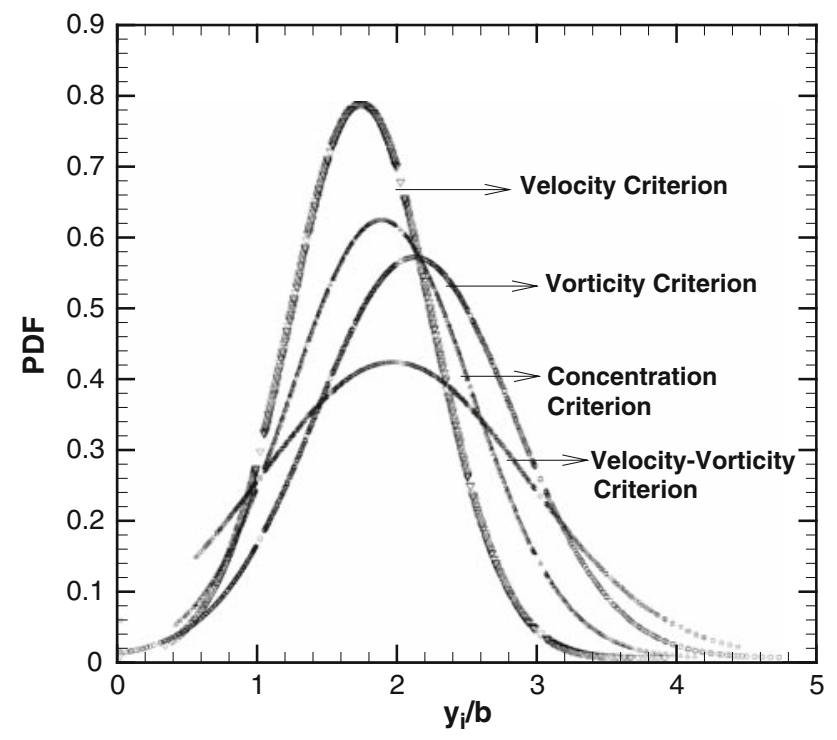

Fig. 11 Probability distribution functions of interface for four different criteria as obtained from DNS data

\section{Discussion}

The instantaneous interface locations (contours) as detected from all criteria stated above are presented in Fig. 10. The interface detected by the Velocity criterion is toward the jet centerline as compared to the other interfaces in most of the region. The interface found from the Vorticity criterion is mostly further away from the jet centerline. The proposed Velocity-Vorticity criterion gives an interface which is sometimes inside and sometimes outside of the Concentration interface. A direct comparison of available criteria has not been demonstrated earlier to the best of our knowledge. The comparison is useful because a good match in the interface would suggest that other criterion could also be used for detection.

\subsection{Probability distribution function (PDF)}

Figure 11 shows the probability distribution function (PDF) of the interface location for DNS results using all the four criteria discussed above. It is observed that all the four curves are approximately Gaussian in nature with mean values $\left(y_{i, \text { mean }} / b\right)$ of $1.86,2.10,1.72$, and 1.95 for Concentration, Vorticity, Velocity, and Velocity-Vorticity criteria, respectively. The corresponding standard deviations are $0.65,0.71,0.51$, and 0.96 , respectively. The value of $\left(y_{i, \text { mean }} / b\right)$ suggests that the maximum probability of finding the interface with Concentration criterion is 1.86 times the local jet width from the jet centerline. The mean and standard deviation obtained here using Concentration criterion compare reasonably well with the reported mean

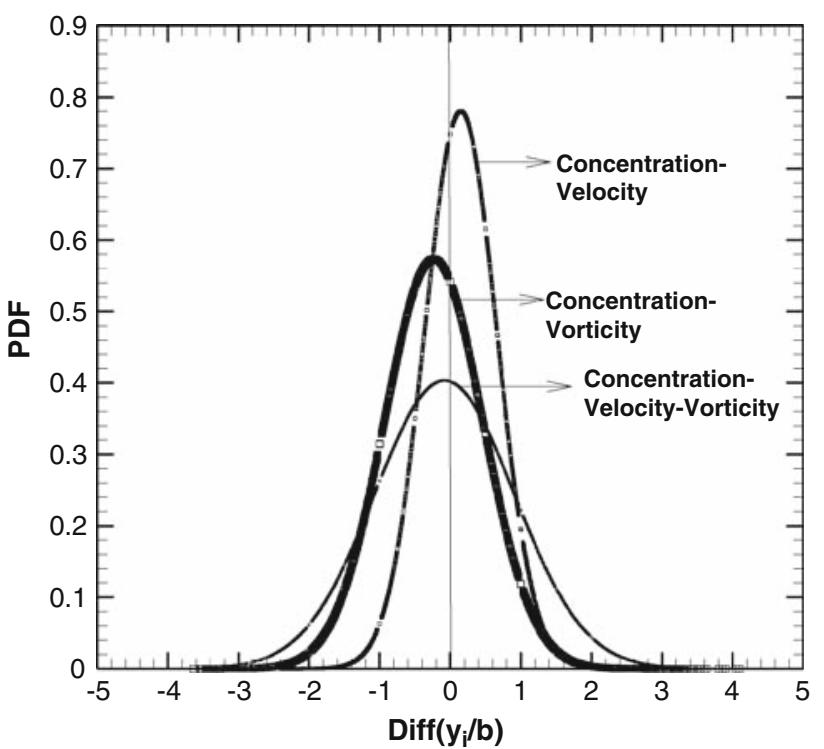

Fig. 12 Probability distribution function of the other three interfaces with respect to the concentration interface as obtained from DNS data

(1.97) and standard deviation (0.41) of Westerweel et al. (2005).

The explanation for the differences observed in the conditionally averaged quantities is explored next. The difference in locations of interface position obtained from Vorticity, Velocity, and Velocity-Vorticity criteria with respect to that obtained from the Concentration criterion's interface are noted. The choice of Concentration criterion for computing the difference is somewhat arbitrary. Note, however, that visually one detects an interface with the help of a passive scalar and the earlier results of Westerweel et al. $(2002,2005)$ employed this criterion; therefore, it has been preferred here. As a matter of fact, the interface obtained here from the Concentration criterion provides only the basis (and is used) for making a comparison against the rest of the criteria. The above treatment is repeated for the entire dataset for each criterion and the probability density function is calculated. A graph is plotted between probability density function and difference between the interface location obtained from those of Concentration criterion and the other criteria- $\operatorname{diff}\left(y_{i} / b\right)$.

A statistical analysis of the resulting approximately Gaussian profiles is shown in Fig. 12. The PDF of the diff $\left(y_{i} / b\right)$ has mean values at $-0.236,0.147$, and -0.0835 for Vorticity, Velocity, and Velocity-Vorticity criteria, respectively. The corresponding standard deviations are $0.696,0.511$, and 0.989 , respectively. It is worth mentioning that although the PDF of interface differences is found to be approximately Gaussian, it does not imply that they should be centered on the concentration interface. Note that the mean value is positive for the Velocity interface for $56.5 \%$ of the time, suggesting that the 
Velocity criterion gives interface more towards the jet centerline as compared to the Concentration interface. This result is consistent with the well-known fact that on an average the velocity width is smaller than the concentration width $\left(b_{\text {conc }} / b_{\text {vel }}=1.2\right)$ where $b_{\text {conc }}$ is the concentration width and $b_{\text {vel }}$ is the velocity width of the jet; see, for example, Agrawal and Prasad 2003b). Similarly, the interface obtained from the Vorticity and Velocity-Vorticity criteria are, respectively, 60.4 and $54.3 \%$ of the time away from the jet centerline as compared to the Concentration interface. The relatively flat nature of the curve for the Velocity-Vorticity criterion suggests that although this criterion will predict substantially different interfacial locations at certain instants, both larger and smaller positions are equally likely.

The results in Figs. 2, 3, and 4 obtained from Vorticity criterion show that their values are overall less as compared to the results of Westerweel et al. (2005). This could be due to the interface being away from the Concentration interface as observed by a noticeable variation in the mean locations. Similarly, the Velocity criterion yields higher values as compared to Westerweel et al. (2005), in particular for velocity and vorticity. This could be attributed to the position of interface being more towards the jet centerline as compared to the benchmark data. Figures 11 and 12 show that, the interface for the Velocity-Vorticity criterion is only slightly away from the jet centerline as compared to the interface from the Concentration criterion. This leads to a slightly smaller value of streamwise velocity (near the jet centerline), cross-stream velocity, and Reynolds shear stress measured for the Velocity-Vorticity criterion as compared to that of Westerweel et al. (2005).

\subsection{Sensitivity of threshold on Velocity-Vorticity criterion}

In this subsection, a sensitivity analysis of the conditionally averaged PIV results obtained from Velocity to Vorticity criterion for different threshold values is presented. The threshold values tested are $0.01,0.03,0.1$, and 0.25 . It is evident from Figs. 13 and 14 that the streamwise velocity and vorticity compare reasonably well against the benchmark data for the three threshold values between 0.01 and 0.1 , and perhaps all values in this range. However, Fig. 15 shows that the Reynolds stress agrees with the benchmark data for threshold values of 0.03 and 0.1 only. Note that a threshold value of 0.25 does not yield acceptable results for any of the quantity considered herein. A similar set of results is obtained with the DNS data.

These results suggest that sub-optimal threshold values (differing by up to a factor of three from the optimal value) may still render acceptable results for the ensemble averaged quantities.

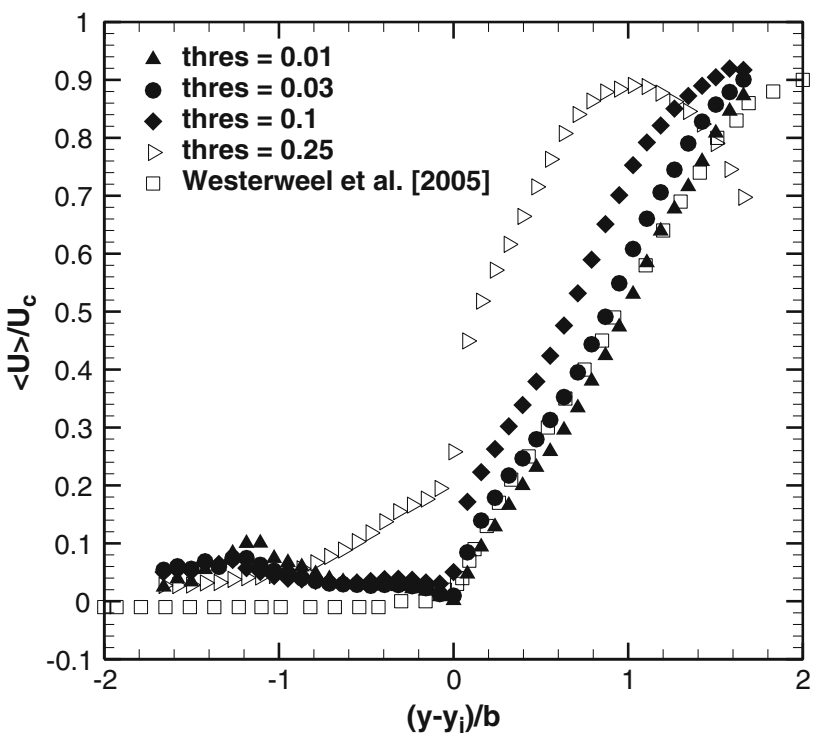

Fig. 13 Conditionally averaged results for streamwise velocity at different threshold levels for PIV data

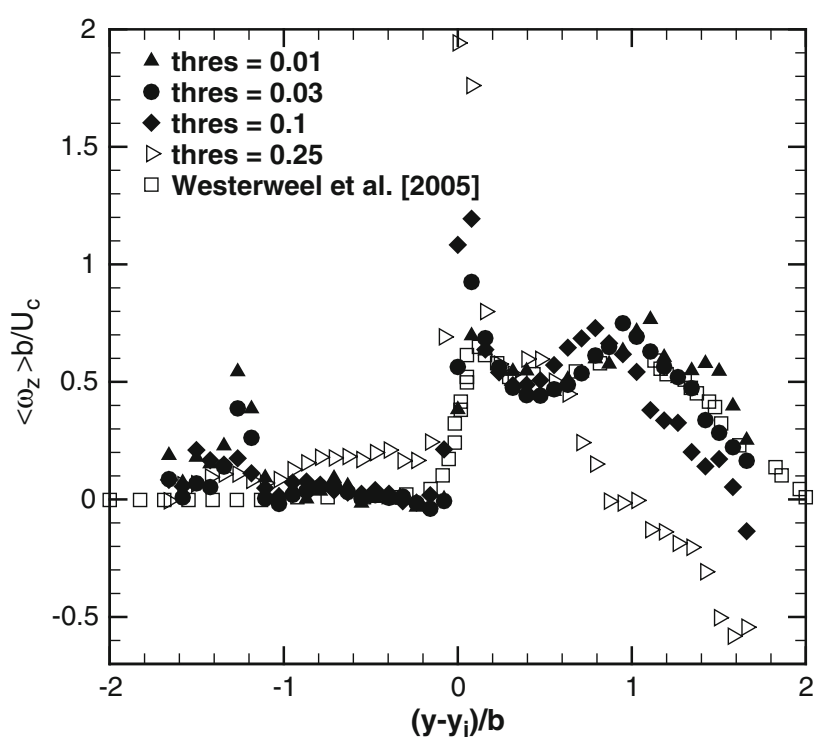

Fig. 14 Conditionally averaged results for normalized azimuthal vorticity across the interface at different threshold values for PIV data

\section{Conclusions}

The different methods for detecting the turbulent/non-turbulent interface proposed in the literature are compared in this work; these include Concentration, Vorticity and Velocity based detection schemes. Towards this end, PIV and DNS data for an axisymmetric turbulent jet have been analyzed. Instantaneous interface and conditionally averaged streamwise velocity, azimuthal vorticity and Reynolds shear stress have been compared against the data 


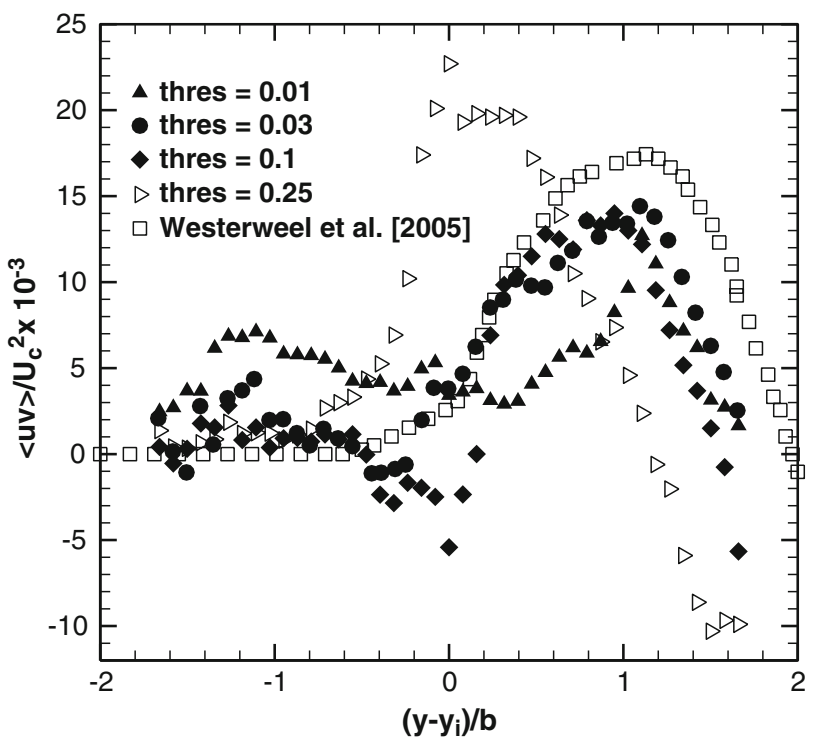

Fig. 15 Conditionally averaged Reynolds stress across the interface at different threshold values for PIV data

of Westerweel et al. (2002, 2005). The availability of threedimensional data and benchmark results in the literature allow for a more meaningful comparison.

A novel method to detect the turbulent/non-turbulent interface from planar wholefield velocity data alone is also proposed. This new criterion is based on a suitable combination of streamwise velocity and azimuthal vorticityquantities which are available or can be easily computed from such data. The proposed criterion is simpler to apply than the other criteria, and yields comparable accuracy at least for the ensemble averaged quantities.

Although the interface results on an instantaneous basis differ substantially for different criteria, the differences are small for the ensemble-averaged quantities. The ensemble-averaged results are also rather insensitive to the value of threshold (with in a range). These results suggest that both conventional quantities like spectrum (Agrawal 2005) and more difficult-to-obtain quantities like conditionally averaged vorticity and Reynolds stress across a turbulent/non-turbulent interface, which were not accessible earlier, can indeed be obtained with particle image velocimetry.

Acknowledgments We are grateful to Professor Jerry Westerweel for his comments and suggestions on an early version of the manuscript. We are also grateful to the two anonymous reviewers for their constructive comments which helped to improve substantially the quality of the manuscript.

Open Access This article is distributed under the terms of the Creative Commons Attribution Noncommercial License which permits any noncommercial use, distribution, and reproduction in any medium, provided the original author(s) and source are credited.

\section{References}

Adrian RJ (2005) Twenty years of particle image velocimetry. Exp Fluids 39:159-169

Agrawal A (2005) Measurement of spectrum with particle image velocimetry. Exp Fluids 39:836-840

Agrawal A, Prasad AK (2002) Properties of vortices in the selfsimilar turbulent jet. Exp Fluids 33:565-577

Agrawal A, Prasad AK (2003a) Measurements within vortex cores in a turbulent jet. J Fluid Eng 125:561-568

Agrawal A, Prasad AK (2003b) Integral solution for the mean flow profiles of turbulent jets, plumes and wakes. J Fluid Eng 125:813-822

Agrawal A, Prasad AK (2004) Evolution of a turbulent jet subjected to volumetric heating. J Fluid Mech 511:95-123

Agrawal A, Djenidi L, Antonia RA (2004) LIF based detection of low-speed streaks. Exp Fluids 36:600-603

Agrawal A, Boersma BJ, Prasad AK (2005) Direct numerical simulation of a turbulent axisymmetric jet with buoyancy induced acceleration. Flow Turbul Combust 73:277-305

Antonia RA (1981) Conditional sampling in turbulence measurements. Annu Rev Fluid Mech 13:131-156

Antonia RA, Prabhu A, Stephenson SE (1975) Conditionally sampled measurements in a heated turbulent jet. J Fluid Mech 72:455480

Bisset DK, Hunt JCR, Rogers MM (2002) The turbulent/non-turbulent interface bounding a far wake. J Fluid Mech 451:383-410

Boersma BJ, Brethouwer G, Nieuwstadt FTM (1998) A numerical investigation on the effect of the inflow conditions on the selfsimilar region of a round jet. Phys Fluids 10:899-909

Chevray R (1982) Entrainment interface in free turbulent shear flows. Prog Energy Combust Sci 8:303-315

Corrsin S (1943) Investigation of flow in an axially symmetric heated jet in air. NACA ACR 3L23 and Wartime Rep. W94

Corrsin S, Kistler AL (1954) The free-stream boundaries of turbulent flows. NACA TN-3133, TR-1244: 1033-1064

Da Silva BC, Pereira JCF (2008) Invariants of the velocity-gradient, rate-of-strain, and rate-of-rotation tensors across the turbulent/ nonturbulent interface in jets. Phys Fluids 20:055101

Dimotakis PE, Maike-Lye RC, Papanotoniou DA (1983) Structure and dynamics of round turbulent jets. Phys Fluids 26:3185-3192

Holzner M, Liberzon A, Gaula M, Tsinober A (2006) Generalized detection of a turbulent front generated by an oscillating grid. Exp Fluids 41:711-719

Holzner M, Liberzon A, Nikitin N, Kinzelbach W, Tsinober A (2007) Small-scale aspects of flows in proximity of the turbulent/ nonturbulent interface. Phys Fluids 19:071702

Holzner M, Liberzon A, Nikitin N, Luthi B, Kinzelbach W, Tsinober A (2008) A Lagrangian investigation of the small-scale features of turbulent entrainment through particle tracking and direct numerical simulation. J Fluid Mech 598:465-475

Hunt JCR, Eames I, Westerweel J (2006) Mechanics of inhomogeneous turbulence and interfacial layers. J Fluid Mech 554:499_ 519

Kibens V, Kovasznay LSG, Oswald LJ (1974) Turbulent-nonturbulent interface detector. Rev Sci Instrum 45:1138-1144

LaRue JC (1974) Detection of the turbulent-nonturbulent interface in slightly heated turbulent shear flows. Phys Fluids 17:1513-1517

LaRue JC, Libby PA (1974) Turbulent fluctuations in the plane turbulent wake. Phys Fluids 17:1956-1967

Lubbers CL, Brethouwer G, Boresma BJ (2001) Simulation of the mixing of a passive scalar in a round turbulent jet. Fluid Dyn Res 28:189-208

Mathew J, Basu AJ (2002) Some characteristics of entrainment at a cylindrical turbulence boundary. Phys Fluids 14:2065-2072 
Pope SB (2000) Turbulent flows. Cambridge University Press, New York

Prasad AK (2000) Particle image velocimetry. Curr Sci 79:101-110

Prasad RR, Sreenivasan KR (1989) Scalar interfaces in digital images of turbulent flows. Exp Fluids 7:259-264

Sreenivasan KR, Ramshankar R, Meneveau C (1989) Mixing, entrainment and fractal dimensions of surfaces in turbulent flows. Proc Royal Soc Lond A 421:79-108
Westerweel J, Hofmann T, Fukushima C, Hunt JCR (2002) The turbulent/non-turbulent interface at the outer boundary of a selfsimilar turbulent jet. Exp Fluids 33:873-878

Westerweel J, Fukushima C, Pedersen JM, Hunt JCR (2005) Mechanics of turbulent-non-turbulent interface of a jet. Phys Rev Lett 95:174501 